\title{
Synthèse
}

\section{Marchés de la pomme de terre dans I'Union européenne}

\section{Anne-Céline Contamine}

Union nationale des producteurs de pommes de terre (UNPT),

43-45, rue de Naples,

75008 Paris

France

<ac.contamine@producteursdepommes deterre.org>

\begin{abstract}
Résumé
Le marché de la pomme de terre européenne se caractérise par des débouchés diversifiés, de la pomme de terre vendue en l'état à la pomme de terre transformée par les agro-industries. Soixante et un million de tonnes de pommes de terre sont produites dans l'Europe des 27 (2005), une production en baisse depuis les années 1960. Derrière ce chiffre, se cachent des réalités bien différentes selon les pays concernés. L'Europe réunit actuellement des pays dont les productions et le commerce agricoles sont bien distincts. Les principaux pays producteurs de pommes de terre, Allemagne, Pays-Bas, France, Royaume-Uni et Belgique, concentrent près de la moitié de la production en volume et $87 \%$ du commerce intra-européen. Les nouveaux pays membres, en pleine restructuration de leur secteur agricole, participent à près de la moitié des surfaces cultivées dans l'Europe des 27, mais seulement à hauteur de $28 \%$ de la valeur de la production européenne. La consommation évolue, la tendance est à la baisse de la consommation de pommes de terre vendues en l'état, alors que la consommation de produits précuisinés de type frite augmente. Une tendance marquée pour les pays les plus développés de l'Europe, mais qui devrait se poursuivre dans les nouveaux pays membres, principalement à l'est de l'Europe. Les industries de transformation sont situées au cœur des bassins de production, majoritairement dans les cinq principaux pays producteurs de pommes de terre. Le développement de futurs bassins de consommation pourrait entraîner leur implantation dans les pays de l'Est.
\end{abstract}

Mots clés : commerce ; consommation alimentaire ; Europe ; production ; Solanum tuberosum.

Thèmes : économie et développement rural ; productions végétales ; transformation ; commercialisation.

\section{Abstract \\ Potato markets in the European Union}

The European potato market has many different prospects: from table potatoes to processed potatoes for the food and the starch industries. Sixty one million potatoes are produced in the 27 member states of the EU (2005), but the production has been declining since the sixties. This global production data does not display the very different economical and agricultural situations. The main producers are Germany, The Netherlands, France, Belgium and the United Kingdom. These 5 countries represent half of the European production and $87 \%$ of intra European trade. Even if the new member states own almost half of the total production area, they are producing only $28 \%$ of the total value of the European potato sector. Potato consumption habits are evolving: the main trend is a decline in fresh consumption and a rise in pre-cooked product consumption. This trend is significant in the developed countries of Europe and should be observed in the new member states in coming years, especially in the countries of Eastern Europe. The food industries are located at the heart of the production areas, mainly in the 5 biggest potato-producing countries. The development of future consumption areas should lead to the establishment of food industry production facilities in the Eastern countries.

Key words: Europe; food consumption; production; trade; Solanum tuberosum.

Subjects: economy and rural development; processing; marketing; vegetal; productions.

Tirés à part : A.-C. Contamine 
e berceau de la pomme de terre est situé dans la cordillère des Andes, où les Incas l'ont cultivée dès 800 à 900 avant JC. Les conquistadores espagnols découvrirent la pomme de terre vers 1550 sur le territoire de l'actuel Pérou et l'introduisirent en Europe à leur retour des Amériques. Trois siècles plus tard, la pomme de terre a toute sa place dans l'alimentation des Européens et des Américains. Au milieu du XIX ${ }^{\mathrm{e}}$ siècle, sa destruction par le champignon pathogène Phytophthora infestans sera une des causes de la grande famine en Irlande, dont le nombre de victimes est estimé entre 500000 et 2,5 millions suivant les sources. Depuis, ce tubercule est cultivé dans le monde entier et consommé de multiples façons. Vendues à l'état frais, les pommes de terre sont également transformées par l'industrie agroalimentaire (frites, purée...). La pomme de terre entre dans de nombreux plats traditionnels, mais également dans de nombreuses préparations industrielles. Un succès incontestable en alimentation mais pas seulement, car la pomme de terre est aussi transformée en fécule de pomme de terre, utilisée par l'industrie papetière, chimique. La production européenne de fécule est régulée par l'Organisation commune de marché, qui définit des quotas de production pour les pays producteurs. Du fait de son organisation originale par rapport aux autres débouchés de la pomme de terre, qui ne sont pas contingentés, cet article ne traitera pas de la pomme de terre féculière.

La pomme de terre est le quatrième produit de récolte consommé dans le monde devant le riz et le maïs ( $\mathrm{kcal} /$ personne par jour). Sa consommation a évolué de façon bien différente suivant les pays. La consommation dans les pays en voie de développement (PED) est passée de $35,3 \mathrm{~g} /$ personne par jour en 1990 à $63,9 \mathrm{~g}$ /personne par jour en 2005, soit une croissance exceptionnelle de $81 \%$. Ce sont dans les pays les moins développés des PED, tels que définis par la Food Agricultural Organisation (FAO), que la consommation a le plus progressé puisqu'elle fait un bond en passant entre 1990 et 2005 de $18,9 \mathrm{~g} /$ personne par jour à $37,7 \mathrm{~g} /$ personne par jour, soit une progression de $99 \%$.

Les quantités consommées dans les pays développés sont bien plus élevées, puisque la consommation est actuellement de 213,7 g/personne par jour. Aujourd'hui pourtant la tendance n'est plus à la croissance, mais à la stabilisation, voire au recul puisqu'en 1990 la consommation des pays développés était de 219,6 g/ personne par jour. Il s'agit donc de deux tendances inversées: la consommation croît dans des pays où la pomme de terre ne faisait pas ou peu partie du régime alimentaire et recule dans des pays historiquement consommateurs.

Le secteur de la pomme de terre a connu de nombreuses évolutions. Jusqu'au début des années 1990, la plupart des pommes de terre étaient cultivées et consommées en Europe, en Amérique du Nord et dans les pays de l'ex-Union soviétique. Depuis lors, la production décline en Europe alors que la production et la demande de pommes de terre ont enregistré une forte croissance en Asie, en Afrique et en Amérique latine. En 2005, pour la première fois, la production de la pomme de terre des PED - 161,5 millions de tonnes environ - a dépassé celle des pays développés (155,9 millions de tonnes).

La Chine est, en 2005, le premier producteur de pommes de terre en volume avec 74 millions de tonnes produites. Depuis les années 1960, la production y a été multipliée par six. La Russie est deuxième producteur mondial avec 37 millions de tonnes et l'Inde suit avec 24 millions de tonnes, soit une production qui a septuplé depuis les années 1960.

Ce qui caractérise la Chine et l'Inde c'est l'explosion des surfaces cultivées, qui ont été multipliées par trois ou quatre depuis 1960, alors que les surfaces des quatre principaux pays européens producteurs ont, quant à elles, diminué de $71 \%$. Dans l'Europe des 27, 2,3 millions d'hectares sont cultivés en 1961 contre 7,9 millions en 1960 (chiffres FAO). En 2005, l'Europe des 27 a produit 61 millions de tonnes de pommes de terre. En réalité, l'Europe administrative et politique des 27 regroupe des pays aux évolutions agricoles et économiques bien différentes.

\section{Production européenne}

En 1961, ce sont plus de 4,5 millions d'hectares qui sont cultivés en pommes de terre à travers l'ensemble des pays qui formeront ensuite l'Europe des 15 (Allemagne, Autriche, Belgique, Danemark, Espagne, Finlande, France, Grèce, Irlande, Italie, Luxembourg, Pays-Bas, Portugal, Royaume-Uni et Suède). Sur les 76,3 millions de tonnes produites, près des quatre cinquièmes le sont par six pays : l'Allemagne, les Pays-Bas, la Belgique, la France, le Royaume-Uni et l'Autriche. À partir des années 1960, la production de l'Europe des 15 baisse au total de $35 \%$ (tableau 1). En réalité à partir des années 1960, la production européenne des pays constituant l'actuelle Europe, tout comme les surfaces, déclinent alors que les rendements progressent (figure 1). À partir de 1990, les pays européens méditerranéens tels que l'Espagne, l'Italie, le Portugal voient leur production reculer fortement. Les productions des pays du nord-ouest européens, dont le climat est plus propice à la production de pommes de terre, subissent des baisses moins importantes, voire une augmentation très importante de leur production, comme cela fut le cas pour la Belgique $(+67 \%)$ et la France $(+38,9 \%)$. On assiste à une concentration importante de la production de pommes de terre dans le nord-ouest de l'Europe.

De plus, l'entrée de l'Espagne et du Portugal dans l'Union européenne en 1986, qui va de pair avec l'ouverture des frontières, a pour résultat de mettre en concurrence les productions locales avec celles des pays du nord-ouest de l'Europe. Les pays du sud de l'Europe se spécialisent notamment dans la production de pommes de terre primeurs pour lesquelles les conditions climatiques sont plus favorables. Ces pommes de terre sont commercialisées dès les premiers jours d'avril. Par la suite, ce sont les primeurs du nord-ouest de l'Europe qui prennent le relais, avant que les cultures principales à partir d'août ne soient récoltées et vendues.

À l'heure actuelle, les cinq principaux pays producteurs européens sont l'Allemagne, sixième producteur mondial (dix millions de tonnes de pommes de terre en 2005 , toutes pommes de terre confondues), les Pays-Bas (6,5 millions de tonnes), l'Angleterre (5,7 millions de tonnes), la France (6,3 millions de tonnes) et la Belgique (2,8 millions de tonnes). Ils totalisent à eux cinq plus de 30 millions de tonnes sur les 43,9 millions de tonnes produites dans l'Europe des 15.

Depuis les années 1990, leur production a peu évolué, elle est de 34,4 millions de tonnes en 1990 et de 33,7 millions de tonne en 2005. En effet, les gains de rendements ont été très importants. Les rendements sont passés de 33 à 42,8 t/ha entre 1990 et 2005 , soit une augmentation de presque $30 \%$, qui a comblé une baisse de surfaces de près de $30 \%$. À l'exception du Danemark, les rendements des autres pays de l'Europe des 15 sont bien plus faibles, 
Tableau 1. Production de pommes de terre de l'Europe des 15 ly compris les pommes de terre destinées aux industries féculières et les plants de pommes de terre).

Table 1. Production of potatoes in the EU-15 (including potatoes for starch and seeds).

\begin{tabular}{|c|c|c|c|c|c|}
\hline Pays & 1961 & $\begin{array}{c}\text { Évolution } \\
\text { entre } 1961 \text { et } 1990\end{array}$ & 1990 & $\begin{array}{c}\text { Évolution } \\
\text { entre } 2005 \text { et } 1990\end{array}$ & 2005 \\
\hline Allemagne & 29945536 & $-51,7 \%$ & 14471000 & $-19,7 \%$ & 11624200 \\
\hline Autriche & 3394825 & $-76,6 \%$ & 793537 & $-3,8 \%$ & 763165 \\
\hline Belgique & 1597330 & $4,2 \%$ & 1664948 & $67,0 \%$ & 2780865 \\
\hline Danemark & 1490089 & $-0,5 \%$ & 1482524 & $6,3 \%$ & 1576400 \\
\hline Espagne & 4918300 & $8,4 \%$ & 5330700 & $-51,9 \%$ & 2563464 \\
\hline Finlande & 1057135 & $-16,6 \%$ & 881400 & $-15,7 \%$ & 742700 \\
\hline France & 14331130 & $-66,8 \%$ & 4754415 & $38,9 \%$ & 6604600 \\
\hline Grèce & 400184 & $138,1 \%$ & 953035 & $-6,5 \%$ & 891063 \\
\hline Irlande & 2145000 & $-71,8 \%$ & 605000 & $-32,4 \%$ & 409200 \\
\hline Italie & 3931500 & $-41,3 \%$ & 2308700 & $-24,0 \%$ & 1753526 \\
\hline Luxembourg & & & & & 19329 \\
\hline Pays-Bas & 3719681 & $89,2 \%$ & 7036000 & $-3,7 \%$ & 6777000 \\
\hline Portugal & 1055596 & $27,2 \%$ & 1343005 & $-57,1 \%$ & 576304 \\
\hline Royaume-Uni & 6811000 & $-5,1 \%$ & 6467000 & $-7,8 \%$ & 5961000 \\
\hline Suède & 1525580 & $-22,3 \%$ & 1186100 & $-20,1 \%$ & 947300 \\
\hline Total Europe des 15 & 76322886 & $-35,4 \%$ & 49277364 & $-10,7 \%$ & 43990116 \\
\hline
\end{tabular}

Source FAO-FAOSTAT (http://faostat.fao.org).

en moyenne de $27 \mathrm{t} /$ ha en 2005. Les gains en rendement résultent de nombreux facteurs. Les techniques culturales se sont améliorées avec des systèmes de rotation des cultures, d'irrigation, du matériel spécialisé et une lutte accrue contre les maladies touchant la plante en culture. De même, la qualité des plants a progressé.
En effet, la recherche variétale a permis une diversification importante des variétés, dans le souci que celles-ci soient plus performantes d'un point de vue des rendements et plus résistantes aux maladies. Actuellement, les techniques de production de plants respectent des normes sanitaires drastiques qui permettent de mieux

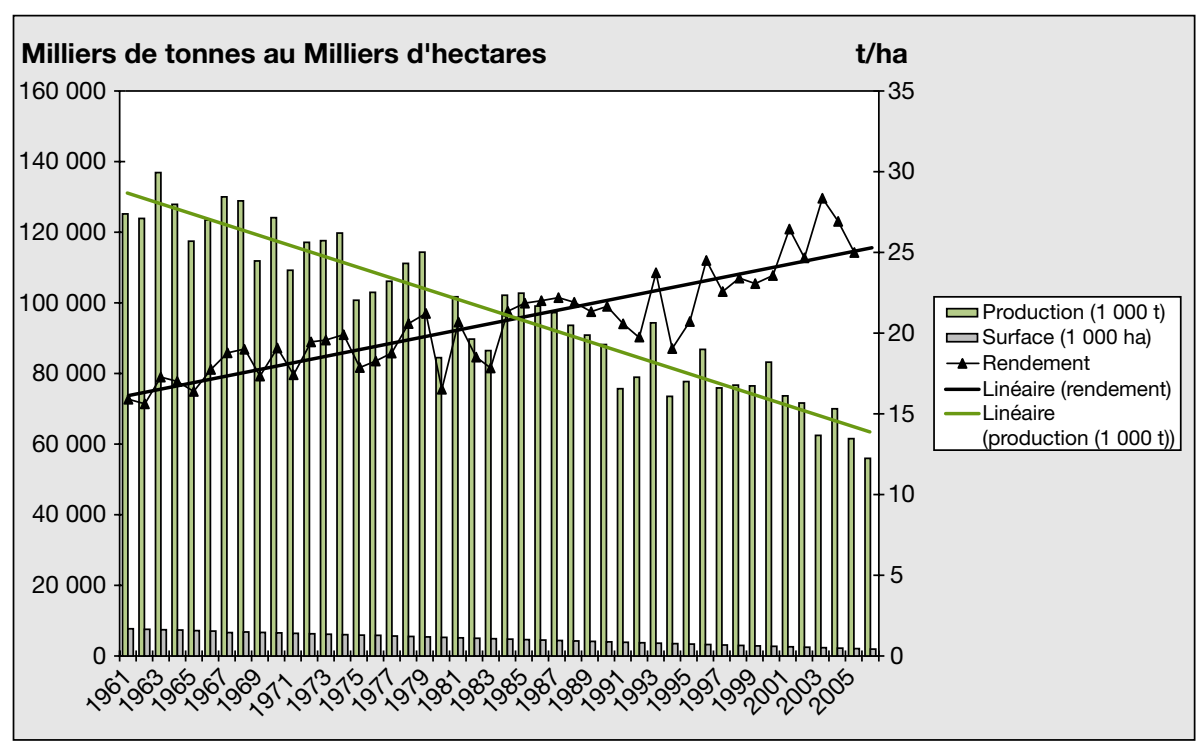

Figure 1. Évolution des surfaces, de la production et des rendements dans l'Europe à 27 (pommes de terre féculières et plants sont inclus).

Figure 1. Evolution of area, production and yield in the EU-27 (including starch and seeds). Source FAO-FAOSTAT (http://faostat.fao.org). maitriser les attaques des ravageurs de même que les viroses. La maitrise de la germination, qui permet que le plant atteigne une qualité optimum lors de la plantation, a également été déterminante pour la progression des rendements.

L'Europe, élargie de 15 à 27 pays membres, produit au total un peu plus de 61 millions de pommes de terre. Un chiffre derrière lequel se cachent des réalités bien différentes selon les pays. L'Europe des 15 regroupe presque les $75 \%$ de la production européenne en volume et en valeur. En effet, la valeur en euros de la production de pomme de terre est évaluée à 9238,1 millions d'euros pour l'ensemble de l'Europe (27 pays). Cette valeur provient pour $72 \%$ de l'Europe des 15 (tableau 2).

Si les nouveaux pays membres ${ }^{1}$ contribuent à près de la moitié des 2,3 millions d'hectares cultivés, ils ne représentent que $29,6 \%$ du total des 61 millions de tonnes produites. Ils contribuent de la même manière à seulement $27,7 \%$ de la valeur européenne de la production.

Par contre, la pomme de terre a un poids important au niveau national pour ces

\footnotetext{
Nouveaux pays membres de l'Europe depuis le $1^{\mathrm{er}}$ mai 2004 : Estonie, Lettonie, Lituanie, Pologne, République Tchèque, Slovaquie, Hongrie, Slovénie, Malte et République de Chypre.
} 


\section{Tableau 2. Importance économique de la production de pommes de terre (pommes de terre féculières et plants sont inclus) en Europe.}

Table 2. Importance of potato production in the European Union (including starch and seeds).

\begin{tabular}{lcccc}
\hline & $\begin{array}{c}\text { Valeur de la production } \\
\text { annuelle } \\
\text { (millions d'euros) }\end{array}$ & 2006-2005 & $\begin{array}{c}\text { Valeur de la production } \\
\text { agricole } \\
\text { (millions d'euros) }\end{array}$ & $\begin{array}{c}\text { Part en valeur } \\
\text { de la production } \\
\text { de pommes de terre } \\
\text { dans la production } \\
\text { agricole totale }\end{array}$ \\
\hline EU 27 & 9238,1 & $30,2 \%$ & $2,9 \%$ \\
EU 25 & 7827,4 & $26,7 \%$ & 300695 & $2,6 \%$ \\
EU 15 & 6675,9 & $29,1 \%$ & 269522,5 & $2,5 \%$ \\
Nouveaux pays membres ${ }^{*}$ & 2562 & $33,2 \%$ & 45995,2 & $5,6 \%$
\end{tabular}

Source Eurostat (http://epp.eurostat.ec.europa.eu).

${ }^{*}$ Nouveaux pays membres de l'Europe depuis le $1^{\text {er }}$ mai 2004 : Estonie, Lettonie, Lituanie, Pologne, République Tchèque, Slovaquie, Hongrie, Slovénie, Malte et République de Chypre.

nouveaux pays membres avec une part en valeur qui représente $5,6 \%$ de la valeur de leur production agricole. Pour les pays de l'Europe des 15, cette part est seulement de $2,5 \%$.

Les données de production des nouveaux pays membres sont peu connues avant les années 2000. La FAO possède les données historiques de production, de surface et de rendement de cinq pays: Bulgarie, Chypre, Malte, Pologne et Roumanie. La baisse des surfaces et de la production a commencé comme pour l'Europe des 15 dans les années 1960, mais à un rythme beaucoup plus lent. C'est à partir des années 1990, que l'on observe les premiers véritables changements avec des baisses de surfaces et de production de respectivement de 57 et $63 \%$ de 1990 à 2005 pour ces cinq pays. Le secteur agricole, qu'il s'agisse de son organisation économique ou de la structure même des exploitations, est en pleine restructuration pour les pays de l'est de l'Europe. Cette restructuration devrait se poursuivre à moyen terme. Depuis l'élargissement de l'Europe, la Pologne est le principal pays producteur de pommes de terre en volume: elle se place à la septième place mondiale avec une production de neuf millions de tonnes. En réalité, on estime que seulement $36 \%$ (Zimnoch et Chotkowski, 2006) de la production totale est commercialisée, le reste étant en grande partie autoconsommée dans les exploitations agricoles polonaises. Avant 2002, seulement $20 \%$ de la production était commercialisée. Une différence de taille par rapport aux pays gros producteurs européens qui valorisent pratiquement toute leur production. Les volumes de pommes de terre utilisés pour l'alimentation animale ont fortement décliné depuis les années 1990, ce qui a d'ailleurs contribué à la baisse de la production totale. Les céréales, dont la culture est plus facile à réaliser et moins coûteuse, ont pu remplacer les pommes de terre (Centro International de la Papa, 2005). La culture de pomme de terre est une des principales cultures polonaises, elle est d'ailleurs cultivée dans toutes les régions du pays. En 2005, les surfaces couvraient 588000 hectares soit 5,3\% de l'ensemble de la surface agricole. Les rendements de ces 25 dernières années d'environ $17,4 \mathrm{t} /$ ha correspondent à près de la moitié des rendements des exploitations allemandes ou néerlandaises. Ces faibles rendements sont dus à plusieurs facteurs : le nombre important des petites parcelles, des techniques culturales sans irrigation et l'utilisation des plants de pommes de terre de qualité médiocre. La pomme de terre polonaise s'exporte pour $90 \%$ vers la Moldavie, la Russie, la Biélorussie et la Bulgarie. L'Europe de l'ouest représente un marché encore peu développé, notamment du fait des normes sanitaires demandées.

Une différence de taille entre les pays de l'Europe des 15 et les pays nouveaux entrants est la taille des exploitations (tableau 3). Dans les cinq principaux pays producteurs, $77 \%$ des surfaces pommes de terre proviennent d'exploitations cultivant plus de 50 ha. On assiste à une concentration des exploitations. Les exploitants se sont professionnalisés et le nombre de petites exploitations a fortement diminué. En France, d'après le ministère de l'Agriculture et de la Pêche, 2005, le nombre d'exploitations pommes de terre a diminué de 5,6\% par an entre 2000 et 2005 pour des emblavements quasiment stables depuis 2000. Cette diminution du nombre des exploitations en pomme de terre de consommation est trois fois plus rapide que celle de l'ensemble des exploitations professionnelles.

\section{Tableau 3. Distribution des exploitations agricoles productrices de pommes de terre selon leur surface totale dans l'Union européenne.}

Table 3. Distribution of potato-growing farms per overall surface size in the EU.

\begin{tabular}{lrrrrrrrr}
\hline \multicolumn{1}{c}{ Hectare } & \multicolumn{1}{c}{} & $\mathbf{>}-\mathbf{2} \mathbf{5}$ & $\mathbf{>} \mathbf{5 - > 1 0}$ & $\mathbf{1 0 - 2 0}$ & $\mathbf{2 0 - 3 0}$ & $\mathbf{3 0 - 5 0}$ & $\mathbf{5 0 - 1 0 0}$ & $\boldsymbol{>} \mathbf{1 0 0}$ \\
\hline Cinq principaux pays producteurs & $0,2 \%$ & $0,6 \%$ & $1,2 \%$ & $3,5 \%$ & $4,7 \%$ & $12,2 \%$ & $26,9 \%$ & $50,7 \%$ \\
Portugal, Espagne, Italie, Grèce & $16,2 \%$ & $16,3 \%$ & $15,2 \%$ & $16,4 \%$ & $8,9 \%$ & $9,5 \%$ & $9,1 \%$ & $8,4 \%$ \\
Europe des 15 & $4,4 \%$ & $4,8 \%$ & $5,2 \%$ & $7,7 \%$ & $6,7 \%$ & $12,2 \%$ & $22,3 \%$ & $36,7 \%$ \\
Nouveaux pays membres* & $19,1 \%$ & $17,1 \%$ & $14,4 \%$ & $12,0 \%$ & $4,5 \%$ & $4,4 \%$ & $5,2 \%$ & $23,3 \%$ \\
\hline
\end{tabular}

Source FAO-FAOSTAT (http://faostat.fao.org).

${ }^{*}$ Nouveaux pays membres de l'Europe depuis le $1^{\text {er }}$ mai 2004 : Estonie, Lettonie, Lituanie, Pologne, République Tchèque, Slovaquie, Hongrie, Slovénie, Malte et République de Chypre. 
La concentration et la spécialisation des exploitations sont une nécessité pour faire face aux investissements importants qui accompagnent la culture de la pomme de terre.

L'Autriche, la Finlande, la Suède, le Danemark, l'Irlande et le Luxembourg présentent une répartition des exploitations pommes de terre suivant la taille totale des exploitations à peu près équivalente à celle observée pour les cinq principaux pays producteurs. Par contre, dans les pays méditerranéens de l'Europe des 15, l'Espagne, la Grèce, l'Italie et le Portugal, plus de $64 \%$ des surfaces pommes de terre sont le fait d'exploitations de moins de 20 ha. Cette répartition de la taille des exploitations pommes de terre rejoint celle des pays nouveaux entrants qui devraient à l'avenir tendre eux aussi vers des exploitations plus importantes et spécialisées. L'Estonie, la Slovaquie, la République tchèque se distinguent avec plus de $50 \%$ des surfaces pommes de terre appartenant à des exploitations de plus de 100 ha.

Les variétés cultivées sont très nombreuses, plus de 1000 sont recensées en Europe. À l'exception de variétés mixtes du type de la variété Bintje, qui peuvent se commercialiser sur le marché du frais comme sur le marché de l'industrie, les variétés sont de plus en plus adaptées aux demandes des consommateurs. Ces demandes varient selon les pays en termes de calibre, de couleur de peau, de texture et de goût. Ainsi, au Royaume-Uni ou en Espagne, les consommateurs apprécient les pommes de terre de gros calibre comme la variété Estima. Ces grosses pommes de terre, appelées bakers en Royaume-Uni, sont consommées garnies d'une préparation. Ce plat jacket potatoes est typique de la consommation anglaise. Le consommateur français est particulièrement attaché au goût. Depuis cinq ans, la filière française a mis en place une segmentation culinaire qui définit les caractéristiques des pommes de terre pour chacun des usages (purée, salade, gratin, frites).

\section{Consommation}

La consommation moyenne d'un Européen est de $217,7 \mathrm{~g} /$ personne par jour. Avec plus de $300 \mathrm{~g}$ /personne par jour, la Lituanie, la Pologne, la Lettonie, le Portugal et le Royaume-Uni sont les plus gros consommateurs de pommes de terre d'Europe.
La consommation de pommes de terre vendues en l'état (également appelées pommes de terre de table) et celle des pommes de terre transformées par l'industrie agroalimentaire ne sont pas distinguées par la FAO. L'analyse des tendances de la consommation repose donc sur des données en provenance des différents pays. La diminution de la consommation de pommes de terre en l'état au profit de produits transformées est la tendance majeure qui caractérise le marché européen. Les produits prépréparés rencontrent un succès grandissant alors que les pommes de terre de table. qui nécessitent une préparation à la maison, voient leur intérêt diminuer. Ce changement de la consommation est plus marqué pour les pays du nord-ouest de l'Europe.

Ainsi en France, la consommation de pomme de terre fraîche baisse ou se maintient difficilement à son niveau depuis plus de dix ans, alors que les ventes de frites ont progressé de $14 \%$ entre 1999 et 2005 (CNIPT, 2006 ; GIPT, 2006).

De même, d'après le ministère allemand, la consommation de pommes de terre de table est passée de 39,0 kg/habitant pour la campagne 2002-2003 (de juillet à juin) à $32,5 \mathrm{~kg} /$ habitant pour la campagne 2003 2004 , alors que la consommation de pommes de terre transformées a augmenté passant de 34,1 à 34,3 kg/habitant.

Pour les pays nouveaux entrants de l'est de l'Europe, le basculement d'une partie de la consommation de pommes de terre de table vers les produits transformés est plus lent, notamment du fait du coût d'achat des produits transformés. En Hongrie, moins de $5 \%$ des pommes de terre sont vendues transformées (Zsom et Kruppa, 2006).

\section{Commerce européen}

Le commerce européen (EU 25) se fait pour $88 \%$ des volumes vers des pays européens (cultures principales, hors plants de pommes de terre et primeurs). Les exportations vers les pays tiers représentent $12 \%$ (d'après COMEXT) pour la saison 2005-2006. Le commerce intraeuropéen est le fait pour $87 \%$ des cinq principaux pays producteurs (Allemagne, France, Royaume-Uni, Pays-Bas, Belgique) entretiennent des relations commerciales très fortes. Pour la campagne 2006-2007, sur les 415000 tonnes importées par la France, 379000 proviennent de la Belgique, de l'Allemagne et des Pays-Bas.

En valeur, les exportations de l'EU 25 représentent en moyenne, pour 2004 et 2005, 69,5 millions d'euros pour les cultures principales et 8,3 millions pour les primeurs. La France est le premier exportateur européen avec 1,8 million de tonnes de pommes de terre exportées pour une valeur de 457 millions d'euros (douanes françaises).

Les importations européennes concernent principalement les primeurs. Celles-ci représentent 168 contre 6,9 millions d'euros pour les importations de cultures principales. Les importations de primeurs proviennent principalement d'Egypte (191 115 de tonnes en 2005-2006), d'Israël (229 565 de tonnes) et du Maroc (32 230 de tonnes). Ce marché commence en février pour atteindre son maximum mi-mars et mi-mai. Ces importations rentrent en compétition avec les primeurs européennes qui rencontrent de plus en plus de problèmes pour s'imposer face aux prix des primeurs importées. Il est à noter que la définition du terme " primeur " a longtemps été floue au niveau international. Ainsi, les primeurs importées sont parfois stockées avant d'être vendues, ce qui n'est pas le cas de la plupart des primeurs européennes, notamment de la région Bretagne en France.

Les importations de primeurs font la jonction entre les vieilles pommes de terre qui ont été stockées depuis leur récolte en août-octobre de l'année précédente avec les premières pommes de terre des cultures de primeurs et hâtives européennes commercialisées à partir de mai (sud) ou de juin (ouest). Les améliorations des conditions de stockage ainsi que l'évolution vers des tubercules dont la peau est adhérente rendent de plus en plus floues la saisonnalité primeurs-cultures principales.

\section{Marché des produits transformés : développement lié au développement de la restauration rapide}

La croissance exceptionnelle de la production de frites depuis les années 1960 d'abord aux États-Unis puis à travers le 
monde, est assez remarquable. Elle est directement liée au développement de la restauration rapide. Les restaurants rapides font leur apparition aux États-Unis dans les années 1950, avec l'implantation des chaînes de restaurants McDonald et Burger King, dont l'expansion se poursuivra tout au long des années 1960 aux États-Unis. C'est seulement dans les années 1970 que ces chaînes conquièrent le Canada, l'Europe de l'Ouest, le Japon, Hong Kong, l'Australie, et la NouvelleZélande.

Dans les années 1980 et 1990, des restaurants de restauration rapide poursuivent leur développement dans le monde entier, notamment pour la première fois en Asie et en Amérique latine (USDA, 2002).

Actuellement, McDonald est présent dans 119 pays avec près de 30000 restaurants (d'après les sites internet de McDonald). Les principaux marchés de l'entreprise restent en dehors des États-Unis, le Japon avec 2400 restaurants sur les 7500 recensés en Asie et l'Europe avec 5500 restaurants pour l'Europe des 15. Dans les nouveaux pays membres (2004), McDonald a une croissance annuelle du nombre de restaurants de $34 \%$. Cette croissance est seulement de 3,5\% aux États-Unis et de $11,5 \%$ pour l'Europe des 15 . Les potentialités de développement de l'industrie se trouvent en Asie et en Amérique latine.

Le développement de l'industrie de transformation de pommes de terre s'est fait en parallèle avec la croissance de la restauration rapide. Cette industrie est apparue dans les années 1940 aux États-Unis. C'est seulement à partir des années 1950, et simultanément au développement de la restauration rapide, qu'elle a connu un véritable développement. Ainsi, la production américaine de pommes de terre surgelées est passée de 58000 tonnes en 1955 à 1,7 million de tonnes en 1980 et est estimée à 4,2 millions de tonnes en 2000 .

Inexistante en Europe avant les années 1960, le développement de l'industrie a suivi l'évolution de la demande. Les industries de transformation sont situées au cour des bassins de production européens et à proximité des principaux consommateurs, soit essentiellement aux Pays-Bas (3,1 millions de tonnes de pommes de terre transformées en 2004), en Allemagne (2,9 millions), en Angleterre ( 2 millions), en Belgique (1,9 million) et en France (1,1 million) (UNPT, 2006). Dans les nouveaux pays entrants, la Pologne (630 000 tonnes) et la République Tchèque (130 000 tonnes) sont les princi- paux producteurs de produits transformés d'après l'Union européenne des industries de transformation de la pomme de terre (UEITP).

Les données sur les produits transformés sont difficiles à obtenir. Les données suivantes proviennent de l'UEITP (UNPT, 2006). On s'attardera sur les cinq principaux pays producteurs: Allemagne, Pays-Bas, Royaume-Uni, Belgique et France.

Les produits précuisinés représentent plus de $70 \%$ des produits transformés. Viennent ensuite les snacks (chips, biscuits apéritifs) [entre 7 et $8 \%$ ] et les produits déshydratés avec un peu plus de $6 \%$. Les Pays-Bas et la Belgique sont les principaux producteurs de produits précuisinés. Á eux deux, ils produisent plus de $66 \%$ du total produit. Soixante-quinze pour cent des produits déshydratés sont fabriqués en Allemagne. Enfin, le Royaume-Uni est le premier producteur de snacks avec 56\% des produits fabriqués.

Les pays du sud de l'Europe, du fait notamment de leurs rendements plus faibles, peuvent difficilement rentrer en compétition avec les cinq principaux pays producteurs pour approvisionner les usines de transformation.

Comme nous l'avons vu précédemment, les industries de transformation sont peu présentes dans les nouveaux états membres, car la consommation de produits finis est encore faible dans ces pays, notamment en l'Europe de l'Est. On peut s'attendre à ce que la consommation suive le même chemin que pour les autres pays européens avec un développement important de la consommation de ces produits. Comme dans les autres cas, les usines de transformation devraient se localiser à proximité de ces bassins de consommation. À l'heure actuelle, les exploitations de ces pays ne sont pas encore prêtes pour répondre aux exigences qualitatives (technologiques), de sécurité alimentaire et phytosanitaire des entreprises de transformation.

Dernièrement, l'industrie de transformation a souffert de la popularité des régimes alimentaires pauvres en graisses. Cette industrie a réagi en développant des produits dont la préparation nécessite l'utilisation de quantité plus faible de graisses. À l'inverse, l'engouement des consommateurs pour des produits précuisinés est très marqué. Ces produits leur évitent une longue préparation non conciliable avec les nouveaux rythmes de vie et les contraintes liées à l'activité professionnelle des deux membres d'un couple.

\section{Échanges mondiaux des produits transformés}

Si le commerce de pommes de terre est en grande majorité intra-européen, les produits finis, tels que les frites, sont échangés au niveau mondial. Sur les trois milliards de dollars américains exportés par les Pays développés, $61 \%$ le sont par l'Europe des 27, largement dominés par les Pays-Bas (898,2 millions de dollars américains) premier exportateur mondial, puis la Belgique (490 millions), la France (190,4 millions), l'Allemagne (95 millions) et le RoyaumeUni (46,8 millions). Le Canada et les ÉtatsUnis représentent $37 \%$ des exportations des pays développés en valeur. La balance commerciale de l'Europe des 27 est positive de 346,2 millions de dollars américains.

\section{Prix}

La production et le commerce des pommes de terre en Europe étant principalement concentrés dans cinq pays européens fortement interdépendants, les prix vont suivre, dans ces pays, des tendances similaires même si les prix ne sont pas identiques.

Une des caractéristiques de la culture de pommes de terre est qu'elle n'est pas subventionnée à l'exception des pommes de terre féculières que nous ne traiterons pas ici. De ce fait, les prix varient très fortement en fonction du jeu de l'offre et de la demande (figure 2).

Ces fortes variations poussent les producteurs à contractualiser une partie de leur production. Suivant les pays, la contractualisation est d'autant plus marquée. Ainsi en Grande-Bretagne, on estime à $80 \%$ de la production à destination du secteur de la transformation agroalimentaire est contractualisée. En France, ce pourcentage est de $65 \%$. Il est estimé à $50 \%$ en Belgique. Les industries sélectionnent des variétés qui sont adaptées à leurs procédés industriels et leur permettent de réduire leurs coûts. Certaines variétés sont exploitées sous monopole par l'industriel. On peut alors presque définir les relations des producteurs qui produisent ces variétés avec les industriels comme des relations d'intégration verticale.

De nouveaux outils de marché, tels que les marchés à terme, voient le jour. Un 


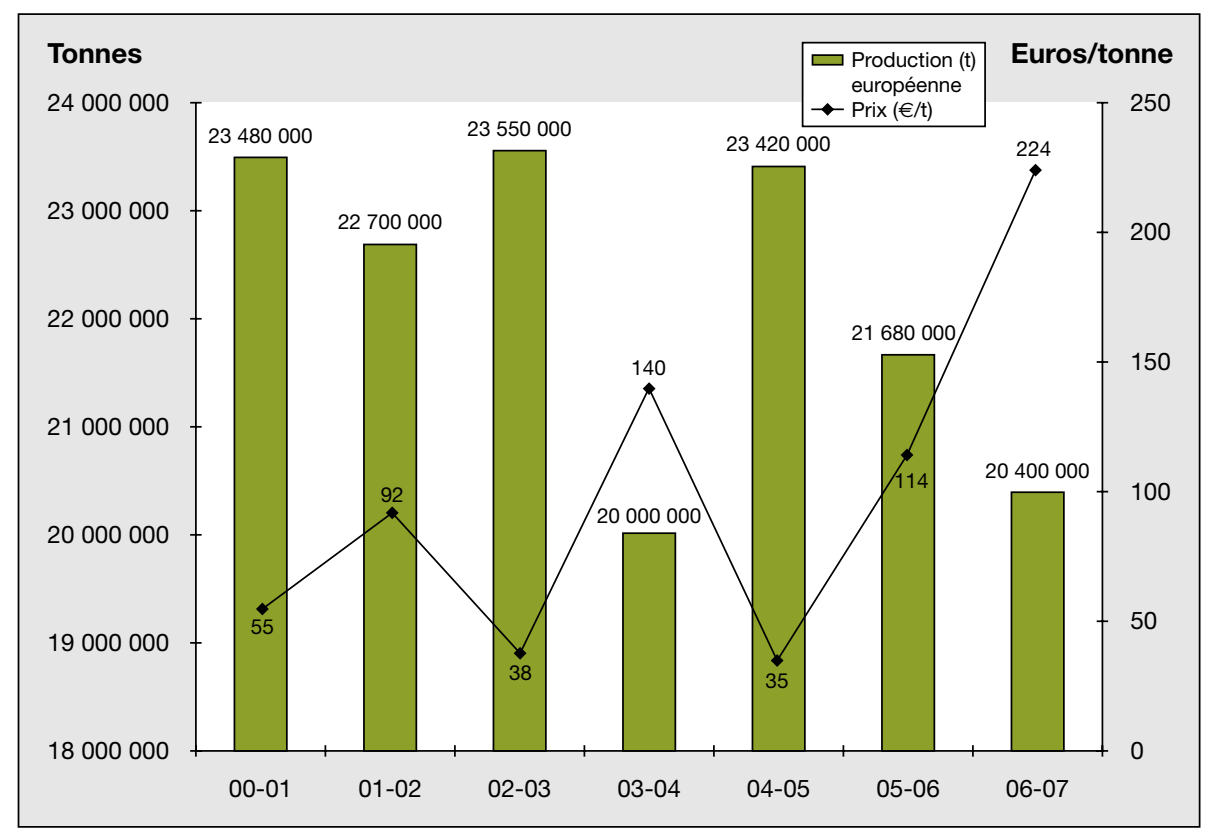

Figure 2. Évolution de la cotation française du marché libre Bintje (SNM) et de la production européenne en volume.

Figure 2. Evolution of the French quotation on the free Bintje market (SNM) and evolution of the European production.

Source : Union nationale des producteurs de pommes de terre (UNPT, 2007) ; élaboré à partir des données du service des nouvelles des marchés (SNM). www.producteursdepommesdeterre.org (rapport d'activité - Congrès 2007).

contrat de marché à terme est un contrat qui stipule la nature, la qualité et la quantité des marchandises ainsi que le lieu et la date de livraison. Les vendeurs et les acheteurs ne s'entendent que sur le prix.

En réalité, les marchandises ne sont jamais livrées. Les obligations de prendre livraison, quand on est acheteur, ou de livrer, lorsqu'on est vendeur, sont évitées par la liquidation des contrats. La combi-

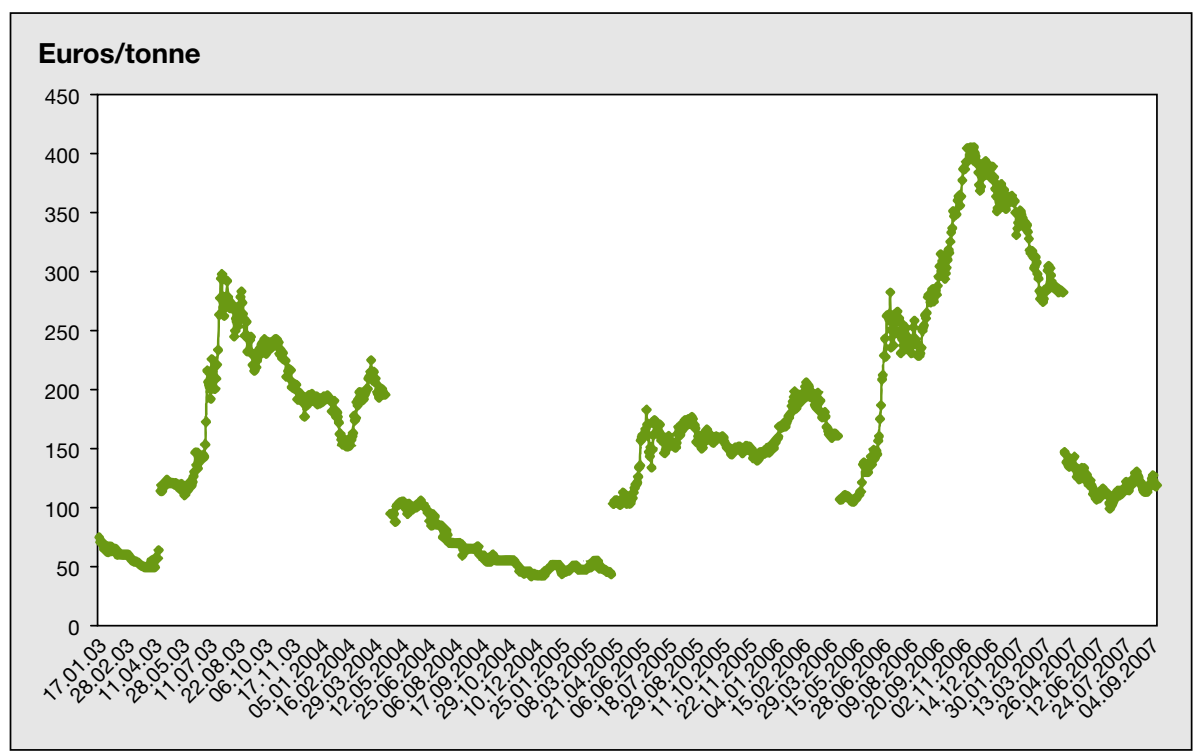

Figure 3. Cotation à terme des pommes de terre à destination industrielle, échéance avril.

Figure 3. Futures prices of processing potatoes, April delivery.

Source RMX - Bourse d'Hanovre : www.wtb-hannover.de. naison des positions sur les marchés à terme et de la vente des pommes de terre sur le marché physique permet alors à l'agriculteur de se couvrir.

La bourse d'Hanovre propose des contrats à terme pour les pommes de terre européennes à destination de l'industrie agroalimentaire. Ces cotations (figure 3) offrent également une visibilité du marché européen, ce qui n'était pas le cas il y a encore cinq ans.

\section{Conclusion}

L'année 2008 est l'année internationale de la pomme de terre. Le choix de ce tubercule par l'Organisation des nations unies est lié aux perspectives de développement de la pomme de terre dans les PED. D'après la FAO, $85 \%$ de la plante est comestible et ses qualités nutritives sont nombreuses: richesse en glucides et en protéines. Cette culture devrait jouer un rôle important dans l'alimentation des populations les plus pauvres.

Le paysage européen de la pomme de terre est contrasté et les défis ne sont pas les mêmes entre les principaux pays européens producteurs et les nouveaux membres de l'est de l'Europe.

Au niveau des cinq principaux pays producteurs, deux défis sont principalement identifiés. Le premier est la lutte contre les maladies bactériennes, fongiques ou dues à la prolifération des insectes. Le mildiou $(P$. infestans) est encore une maladie qui affecte régulièrement les cultures et la production. L'amélioration variétale est une des pistes suivies pour développer des variétés qui résistent mieux aux attaques des organismes pathogènes. Une autre piste fait l'actualité, celle des organismes génétiquement modifiés.

Un autre défi de taille : répondre à l'évolution des habitudes de consommation. Les consommateurs européens souhaitent des produits faciles à préparer, qui n'affectent pas leur santé, et dont les méthodes de production respectent l'environnement. Si la culture de pomme de terre répond déjà à une partie de ces demandes, les possibilités d'innovation sont nombreuses, que cela soit dans les méthodes de production ou dans les produits finis.

Les nouveaux entrants de l'Europe de l'Est sont potentiellement d'importants producteurs de pommes de terre. Néanmoins, leur secteur agricole, en pleine mutation, n'est pas encore prêt à répondre aux exigences qualitatives et logistiques du mar- 
ché européen. L'adaptation des variétés, la qualité des plants et l'amélioration des systèmes de production avec notamment le développement de l'irrigation, et l'utilisation de matériel adapté devraient permettre à ces productions de progresser d'un point de vue qualitatif. D'ici 20 ans, la production européenne pourrait nous présenter un tout nouveau visage.

\section{Références}

Centro Internacional de la Papa. Situation actuelle et perspective de l'économie mondiale de pomme de terre. In : La pomme de terre dans les années 1990. Rome: Food and Agriculture Organisation (FAO), 2005.

Comité national interprofessionnel de la pomme de terre (CNIPT). Rapport d'activité 2006. Paris: CNIPT, 2006. www.cnipt.com [accédé en septembre 2007].

Groupement interprofessionnel pour la valorisation de la pomme de terre (GIPT). Rapport d'activité 2006. Paris: GIPT, 2006. www.gipt.net [accédé en septembre 2007].

Ministère de l'Agriculture et de la Pêche, Agreste. Enquête structure 2005. Paris: ministère de l'Agriculture et de la Pêche, 2005. http ://agreste.agriculture.gouv.fr [accédé en mars 2008].

Union nationale des producteurs de pommes de terre (UNPT). Rapport d'activité 2007. Paris : UNPT, 2007. www.producteursdepommesdeterre.org [accédé en septembre 2007].
Union nationale des producteurs de pommes de terre (UNPT). Compte rendu du Congrès 2006. Paris : UNPT, 2006. www. producteursdepommesdeterre.org [accédé en septembre 2007].

United States Department of Agriculture (USDA), Economic Research Service. French fries driving globalization of frozen potato industry. Agricultural Outlook 2002(October): 8-11; try. Agricultural Outloc gov/publications/agoutlook/oct2002/ao295c.pdf.

Zimnoch Guzowwka E, Chotkowski J. Potato sector in Poland. In : Haase NU, Haverkort AJ, eds. Potato developments in a changing Europe. Wageningen (The Netherlands): Wageningen Academic Publishers, 2006.

Zsom E, Kruppa J. Current trends in the Hungarian potato production. In : Haase NU, Haverkort AJ, eds. Potato developments in a changing Europe. Wageningen (The Netherlands) : Wageningen Academic Publishers, 2006. 